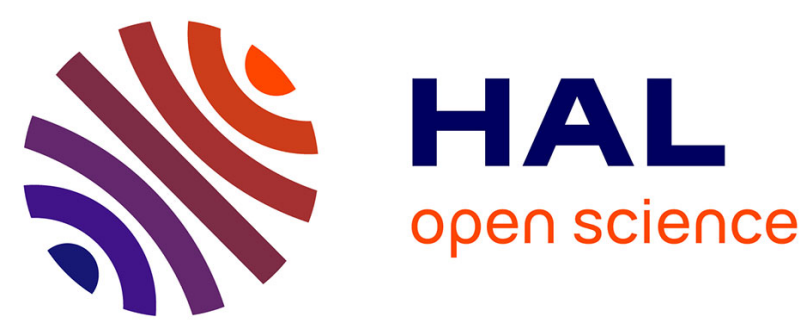

\title{
Second order sliding-mode observer for estimation of vehicle dynamic parameters
}

\author{
N. M'Sirdi, N. Rabhi, A. Fridman, L Davila, J Delanne
}

\section{To cite this version:}

N. M'Sirdi, N. Rabhi, A. Fridman, L Davila, J Delanne. Second order sliding-mode observer for estimation of vehicle dynamic parameters. International Journal of Vehicle Design, 2008, 48, pp.190 - 207. 10.1504/IJVD.2008.022576 . hal-01966653

\section{HAL Id: hal-01966653 \\ https://hal-amu.archives-ouvertes.fr/hal-01966653}

Submitted on 29 Dec 2018

HAL is a multi-disciplinary open access archive for the deposit and dissemination of scientific research documents, whether they are published or not. The documents may come from teaching and research institutions in France or abroad, or from public or private research centers.
L'archive ouverte pluridisciplinaire HAL, est destinée au dépôt et à la diffusion de documents scientifiques de niveau recherche, publiés ou non, émanant des établissements d'enseignement et de recherche français ou étrangers, des laboratoires publics ou privés. 


\title{
Second order sliding-mode observer for estimation of vehicle dynamic parameters
}

\author{
N.K. M'Sirdi* and A. Rabhi \\ LSIS, CNRS UMR 6168, \\ Dom. Univ. St. Jérôme, Av. Escadrille Normandie - Niemen 13397, \\ Marseille Cedex 20, France \\ E-mail: msirdin@1sis.org \\ E-mail: abdelhamid.rabhi@u-picardie.f \\ ${ }^{*}$ Corresponding author
}

\section{Fridman and J. Davila}

Department of Control and Robotics,

Division of Electrical Engineering,Faculty of Engineering,

Universidad Nacional Autonoma de Mexico,

Ciudad Universitaria, 04510, D.F., Mexico

E-mail: 1fridman@unam.mx

E-mail: jadavila@unam.mx

\section{Y. Delanne}

LCPC Nantes:

Division ESAR BP 4434144 Bouguenais Cedex, France

E-mail: yves.delanne@lcpc.fr

\begin{abstract}
This paper uses second-order sliding mode observers to build up an estimation scheme allowing to identify the tyre longitudinal equivalent stiffness and the effective wheel radius using the existing ABS angular sensors. This estimation strategy, based on use of the proposed observer could be used with data acquired experimentally to identify the longitudinal stiffness and effective radius of vehicle tyres. The actual results show effectiveness and robustness of the proposed method.
\end{abstract}

Keywords: sliding modes; nonlinear observers; robust state and stiffness estimation; wheel slip estimation.

Reference to this paper should be made as follows: M'Sirdi, N.K., Rabhi, A., Fridman, L., Davila, J. and Delanne, Y. (2008) 'Second order sliding-mode observer for estimation of vehicle dynamic parameters', Int. J. Vehicle Design, Vol. 48, Nos. 3/4, pp.190-207.

Biographical notes: Nacer K. M'Sirdi is Professor at Polytech Marseille and University Marseille III. He got PhD in Electronics and the Doctorat d'Etat in adaptive signal processing for non stationary signals at the INPG in 1988. He was Assistant Professor in University of Paris 6 in 1987 and Professor at University of Versailles in 1993 and dean of Laboratoire de Robotique de Versailles from 2000 to 2004. From 2005, he is member of LSIS. His main research activities deal with adaptive and robust control, signal processing, diagnosis and observation for complex systems such as vehicles and robots. 
Abdelhamid Rabhi received the Master Degree from the University of Versailles, in robotics and control systems. He got his PhD in observation and control for non-linear and complex systems in December 2006. $\mathrm{He}$ is interested in applications for the vehicle dynamics.

Leonid Fridman received his MS in Mathematics from Kuibyshev (Samara) State University, Russia, PhD in Applied Mathematics from Institute of Control Science (Moscow), and DSc in Control Science from Moscow State University of Mathematics and Electronics in 1976, 1988, 1998 correspondingly. In 2002, he joined the Department of Control and Robotics, Division of Electrical Engineering, Engineering Faculty, National of Autonomous University of Mexico, Mexico. His research interests include variable structure systems, nonlinear observation, singular perturbations, systems with delay. He is editor of three books, five special issues on sliding modes and published over 200 technical papers.

Jorge Davila received the $\mathrm{MSc}$ and $\mathrm{PhD}$ from the National Autonomous University of Mexico (UNAM) in 2004 and 2008 respectively. His professional activities have been concentrated in the observation of linear and nonlinear systems with unknown inputs, nonlinear observation theory, high-order sliding-mode control and its applications.

Yves Delanne received his Doctorat in Department of Mecanics and Acoustics of INSA of Lyon in 1976. He was Dean of Acoustics and Signal Processing Division of the LCPC. Then he was chief of the section 'Surface des chaussées et dynamique des véhicules' and as D.R. (Research Director) in LCPC (Laboratory of 'Ponts et Chaussés', center of Nantes) he was the dean division ESAR Division (ESAR: Entertainment, Security and Acoustic of Roads). He received his HDR (Habiltation à Diriger des Recherche) from University of Versailles (2004).

\section{Introduction}

Car accidents occur for several reasons which may involve the driver or components of the vehicle or environment. Such situations appears when the vehicle is driven beyond the adherence or stability limits. However new active safety systems are developed, improved, and installed on vehicles for real-time monitoring and controlling the dynamic stability (Electronic Braking Systems (EBS), Anti-lock Braking Systems (ABS), Electronic Stability Program (ESP)). The active safety becomes more important in recent research on Intelligent Transportation Systems (ITS) technology. Nevertheless, the possibility of rectifying an unstable condition can be compromised by physical limits. Therefore, it is extremely important to detect (on time) a tendency towards instability. This has to be done without adding expensive sensors, so it requires quite robust observers looking forward based on the physics of interacting systems (the vehicle, the driver and the road).

The tyre forces properties affect the vehicle dynamic performance. The control of ground - vehicle interactions becomes important due to research efforts on intelligent transportation systems, and specially, on automated highway systems. The design of traction controller is based on the assumption that vehicle and wheel angular velocities 
are both available online by direct measurements and/or estimations. Thus the knowledge of tyre parameters and variables (stiffness, forces, velocities, wheel slip and radius) is essential to advanced vehicle control systems such as ABS, Traction Control Systems (TCS) and ESP (M'Sirdi et al., 2007, 2008). However, tyre forces and road friction are difficult to measure directly and to represent precisely by some deterministic model equations. In the literature, their values are often deduced by some experimentally approximated models (Samadi and Nikravesh, 1999; Dugoff and Segel, 1970; Bakker et al., 1989). This work is focused to the online estimation of the tyres sleep, adherence, stiffness and effective radius. The vehicle state is estimated and the tyre forces are identified (M'Sirdi et al., 2008). The main contribution is the robust online estimation of the tyre effective radius, wheel sleep and velocities, needed for a control, by using only simple low cost sensors (ABS sensors).

Recently, many analytical and experimental studies have been performed on estimation of the frictions and contact forces between tyres and road (Ray, 1995; Rabhi et al., 2004b; Gustafsson, 1997). Tyre forces can be represented by the nonlinear (stochastic) functions of wheel slip. The deterministic tyre models encountered are complicated and depend on several factors (as load, tyre pressure, environmental characteristics, etc.) (Dugoff and Segel, 1970; Pacejka and Besseling, 1997; Clover and Bernard, 1998). This makes online estimation of forces and parameters difficult for vehicle control applications and detection and diagnosis for driving monitoring and surveillance (Rabhi et al., 2003). In Drakunov et al. (1995), Canudas et al. (2003) and Rabhi et al. (2003), application of sliding mode control is proposed. Observers based on the sliding mode approach have been also used in Rabhi et al. (2004a). In Ray (1995) an estimation based on least squares method and Kalman filtering is applied for estimation of contact forces. Gustafsson (1997) presented a tyre/road friction estimation method based on Kalman filter to give a relevant estimates of the slope of $\mu$ vs. slip $(\lambda)$, that is, the relative difference in wheel velocity. The paper by Carlson and Gerdes (2003) presented an estimator for longitudinal stiffness and wheel effective radius using vehicle sensors and Global Positioning System (GPS) for low values of slip.

Observers robust to unknown inputs are efficient for estimation of road profile and the contact forces (Rabhi et al., 2004a). Acceleration and braking manoeuvres modify the wheel slip. This phenomenon could be controlled by means of its regulation while using sliding mode approach (M'Sirdi et al., 2004; Rabhi et al., 2004a). This method enhances the road safety leading to better vehicle adherence and manoeuvrability but the vehicle controllability in its environment along the road admissible trajectories still remain an important open problem.

From the other hand, it is necessary to remark that observers for mechanical systems with unknown inputs based on standard first order sliding mode approach (as for example, Barbot et al., 2002; Bartolini et al., 2003; Pisano and Usai, 2004) has the following disadvantages:

- for observation of the velocity a filtering is needed corrupting the results

- the need of filtering in the observation process destroys the finite time convergence property, and the separation principle must be taken into account to design a control

- for the uncertainties and parameters identification a second filtering is necessary.

This leads to a bigger distortion of results. 
A robust exact differentiator (Levant, 1998) based on super twisting algorithm (Levant, 1993) ensures a finite time convergence to the values of the corresponding derivatives and provides the best possible accuracy of the derivatives for the given value even considering deterministic noise, sampling step and in the case of discrete measurements.

In this paper, a nominal model of the vehicle is considered and the the super-twisting based robust exact observer (Davila et al., 2005) is applied for estimation of rotational velocities. The stiffness and effective radius are identified by application of a dynamical identification algorithm. The robust exact observer (Davila et al., 2005; Davila et al., 2006) used in this paper allows

- to make the velocity observation without filtering

- to provide finite time convergence to the exact value of the rotational velocity, ensuring separation principle

- to identify the uncertainties with only just one filtering

- to apply a continuous time parameter identification algorithm for system parameters identification.

This work deals with a simple vehicle model coupled with wheel - road contact. It is proposed a vehicle model for the online estimation using robust observers. The main characteristics of the vehicle longitudinal dynamics were taken into account in the developed model. The obtained dynamics equations may be written in a state space allowing to define an observer based on the sliding mode approach (as presented in M'Sirdi et al., 2004; Rabhi et al., 2004a). The observer has been used to reconstruct the global system state components and then to estimate the tyres forces (M'Sirdi et al., 2004; Rabhi et al., 2004a). The use of sliding mode approach has been motivated by its robustness with respect to the parameters and modelling errors and has been shown to cope well with this problem.

Figure 1 Wheel dynamics and the ABS system (see online version for colours)

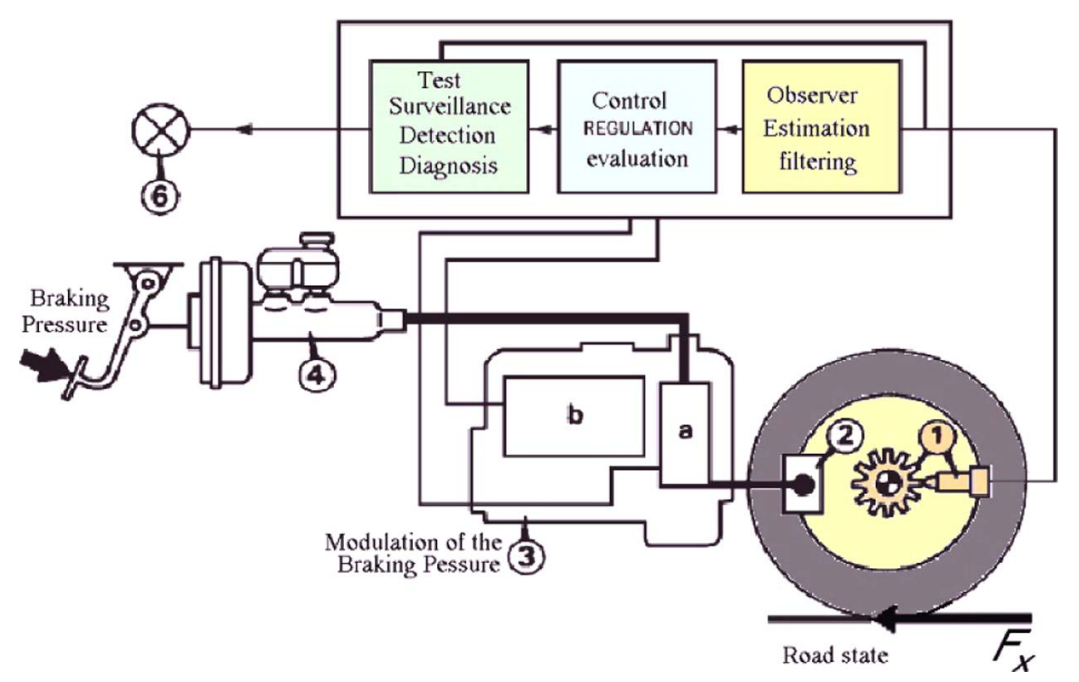


This paper presents a method to estimate the wheel angular velocities by considering the wheel angular position measurements (produced by an ABS variable reluctance sensor as shown in Figures 1 and 2). As a second step, we estimate the longitudinal stiffness and wheel effective radius using additional sensors for the accelerating torque and the linear velocity of the vehicle. The proposed method of estimation is verified through one-wheel simulation model with a 'Magic formula' tyre model and then application results (on a Peugeot 406) show an excellent reconstruction of the velocities, tyre forces and radius estimation.

The developed estimations can be used to detect critical driving situations and then improve the security. It can be used also in several vehicle control systems such as ABS, TCS, diagnosis systems, etc.

\section{Problem statement}

Consider the simplified motion dynamics of a quarter-vehicle model, capturing only nominal behaviour. This model retains the main characteristics of the longitudinal dynamic. For a global application, this method can be easily extended to the complete vehicle and involve the four wheels.

Applying Newton's law to wheel and vehicle dynamics, the equations of nominal motion are given by

$$
\begin{aligned}
& \dot{\theta}=\omega \\
& J \dot{\omega}=J \ddot{\theta}=T_{f}-R_{e} F_{x} \\
& m \dot{v}_{x}=F_{x}
\end{aligned}
$$

where $m$ is the vehicle mass and $J, R_{e}$ are the inertia and effective radius of the tyre, respectively. $v_{x}$ is the linear velocity of the vehicle, $\theta$ is the angular position of the considered wheel, $\omega$ is the angular velocity of the considered wheel, $T_{f}$ is the accelerating (or braking) torque, and $F_{x}$ is the tyre/road friction force. The tractive (respectively braking) force, produced at the tyre/road interface when a driving (braking) torque is applied to a pneumatic tyre, has an opposed direction to relative motion between the tyre and road surface. This relative motion determines the tyre slip properties. The wheel - slip is due to deflection in the contact patch. The longitudinal wheel-slip $\lambda$ is generally called the slip ratio and can be described by a kinematic relation like (Pacejka and Besseling, 1997; Bakker et al., 1989):

$$
\left\{\begin{array}{l}
\lambda=\frac{R_{e f} \omega}{v_{x}}-1 \quad \text { if } v_{x}>R_{e f} \omega \text { (braking) } \\
\lambda=1-\frac{R_{e f} \omega}{v_{x}} \text { if } v_{x}<R_{e f} \omega \text { (traction) }
\end{array}\right.
$$

During ordinary driving, however, the tyre slip rarely exceeds $5 \%$. By linearising the model in a small region (around origin), the force slip relation can be characterised as follows (see Figure 2)

$$
F_{x}=C_{x}\left(\frac{v_{x}-R_{e} \omega}{v_{x}}\right)
$$


where $F_{x}$ and $C_{x}$ are, respectively, the force and the longitudinal stiffness of the tyre(s). The dynamic equation of the whole system can be written in state space form by defining the following state variables. The angular position $x_{1}=\theta$ is measured by the ABS sensor. The angular velocity $x_{2}=\dot{\theta}=\omega$ is not measured and can be obtained by observer application. The vehicle velocity $x_{3}=v_{x}$, and the accelerating torque $u=T_{f}$ are assumed measurable. Note that these expressions assume that velocity is non zero by definition. We can write the system model

$$
\begin{aligned}
& \dot{x}_{1}=x_{2} \\
& \dot{x}_{2}=\frac{u}{J}-\frac{R_{e} C_{x}}{J}+\frac{R_{e}^{2} C_{x}}{J} \frac{x_{2}}{x_{3}} \\
& \dot{x}_{3}=\frac{C_{x}}{m}-\frac{C_{x} R_{e}}{m} \frac{x_{2}}{x_{3}} \\
& y=\left[\begin{array}{cc}
x_{1} & 0 \\
0 & x_{3}
\end{array}\right] .
\end{aligned}
$$

The task is to reconstruct the angular velocity $\left(x_{2}\right)$ of the system by using $x_{1}$ and $u$. The equivalent output injection will be used for parameters identification. An auxiliary system will be introduced for the variable $x_{3}$ in order to obtain an equivalent output injection for this variable.

Figure 2 Wheel slip - forces steady state characteristics (see online version for colours)
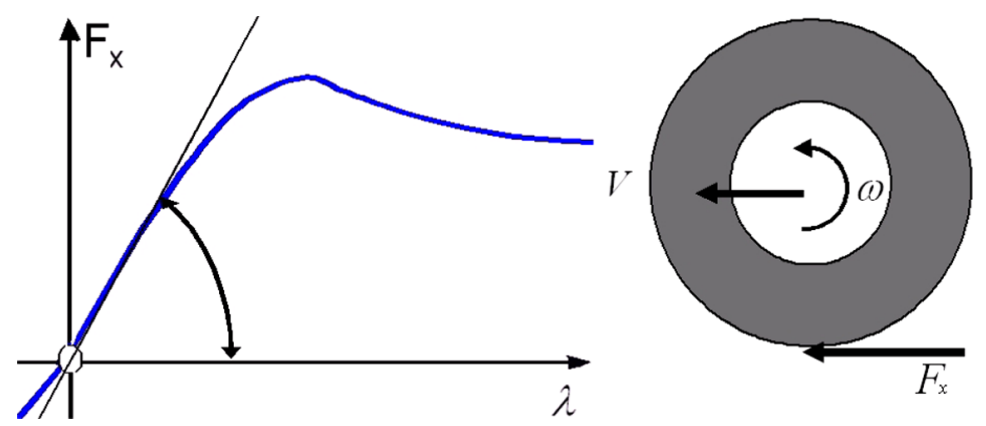

\section{State observation}

\subsection{States $x_{1}, x_{2}$}

Consider the subsystem with state variables $x_{1}=\theta, x_{2}=\dot{\theta}=\omega$, and the control input $u=T_{f}$ (may be computed in function of the system states or their estimates), this submodel of (6) can be rewritten in the state space form as follows:

$$
\begin{aligned}
& \dot{x}_{1}=x_{2}, \\
& \dot{x}_{2}=f_{1}\left(t, x_{1}, x_{2}, u\right)+\xi_{1}\left(t, x_{1}, x_{2}, u\right), \\
& y=x_{1},
\end{aligned}
$$

where the nominal part of the system dynamics is represented by $f_{1}\left(t, x_{1}, x_{2}, u\right)=\frac{u}{J}$ containing the known nominal functions, while uncertainties are concentrated in 
the term $\xi_{1}\left(t, x_{1}, x_{2}, u\right)=-\frac{R_{e} C_{x}}{J}+\frac{R_{e}^{2} C_{x}}{J}$. The system (7), understood in Filippov's sense (Filippov, 1988) is assumed such that the functions $f_{1}\left(t, x_{1}, x_{2}, u\right)$ and the perturbation $\xi_{1}\left(t, x_{1}, x_{2}, u\right)$ are Lebesgue-measurable and uniformly bounded in any compact region of the state space.

Our task is then to design a finite-time convergent observer of the angular velocity $x_{2}=\dot{\theta}=\omega$ assuming that the position $x_{1}=\theta$, the torque $u$, and the nominal model are available. Only the scalar case $x_{1}, x_{2} \in R$ is considered for simplicity. In general case the observers are constructed in exactly the same way for each wheel position variable $x_{1 j}$ in parallel.

The proposed super-twisting observer for the system (7) takes the form Davila et al. (2005)

$$
\begin{aligned}
& \dot{\hat{x}}_{1}=\hat{x}_{2}+z_{1} \\
& \dot{\hat{x}}_{2}=f_{1}\left(t, x_{1}, \hat{x}_{2}, u\right)+z_{2}
\end{aligned}
$$

where $\hat{x}_{1}$ and $\hat{x}_{2}$ are the state estimations, and the correction variables $z_{1}$ and $z_{2}$ are calculated by the super-twisting algorithm

$$
\begin{aligned}
& z_{1}=\lambda\left|x_{1}-\hat{x}_{1}\right|^{1 / 2} \operatorname{sign}\left(x_{1}-\hat{x}_{1}\right) \\
& z_{2}=\alpha \operatorname{sign}\left(x_{1}-\hat{x}_{1}\right) .
\end{aligned}
$$

It is taken for ensures observer convergence that at the initial moment $\hat{x}_{1}=x_{1}$ and $\hat{x}_{2}=0$.

Taking $\tilde{x}_{1}=x_{1}-\hat{x}_{1}$ and $\tilde{x}_{2}=x_{2}-\hat{x}_{2}$ we obtain the error equations

$$
\begin{aligned}
& \dot{\tilde{x}}_{1}=\tilde{x}_{2}-\lambda\left|\tilde{x}_{1}\right|^{1 / 2} \operatorname{sign}\left(\tilde{x}_{1}\right) \\
& \dot{\tilde{x}}_{2}=F\left(t, x_{1}, x_{2}, \hat{x}_{2}\right)-\alpha \operatorname{sign}\left(\tilde{x}_{1}\right)
\end{aligned}
$$

where $F\left(t, x_{1}, x_{2}, \hat{x}_{2}\right)=f_{1}\left(t, x_{1}, x_{2}, u\right)-f_{1}\left(t, x_{1}, \hat{x}_{2}, u\right)+\xi_{1}\left(t, x_{1}, x_{2}, u\right)$. In our case, the system states are bounded, then the existence of a constant $f^{+}$is ensured such that

$$
\left|F\left(t, x_{1}, x_{2}, \hat{x}_{2}\right)\right|<f^{+}
$$

holds for any possible $t, x_{1}, x_{2}$ and $\left|\hat{x}_{2}\right| \leq 2 \sup \left|x_{2}\right|$. The state boundedness is true, because the system (7) is BIBS (Bounded Input - Bounded State) stable, and the control input $u=T_{f}$ is bounded. The maximal possible acceleration in the system is a priori known and it coincides with the bound $f^{+}$. Let $\alpha$ and $\lambda$ satisfy the following inequalities, where $p$ is some chosen constant, $0<p<1$.

$$
\begin{aligned}
& \alpha>f^{+}, \\
& \lambda>\sqrt{\frac{2}{\alpha-f^{+}}} \frac{\left(\alpha+f^{+}\right)(1+p)}{(1-p)},
\end{aligned}
$$


Theorem 3.1 (Davila et al., 2005): Suppose that condition (11) holds for system (7), and the parameters of the observer (8) are selected according to (12). Then, the observer (8) guarantees the convergence of the estimated states $(\hat{x}, \dot{\hat{x}})$ to the real value of the states $(x, \dot{x})$ after a finite time transient, and there exists a time constant $t_{0}$ such that for all $t \geq t_{0},\left(\hat{x}_{1}, \hat{x}_{2}\right)=\left(x_{1}, x_{2}\right)$.

The proof of this theorem is presented in the work Davila et al. (2005).

Let $f_{1}, x, z_{1}, z_{2}$ be measured at discrete times with the time interval $\delta$, and let $t_{i}$, $t_{i+1}$ be successive measurement times. Consider a discrete modification of the observer (the Euler scheme)

$$
\begin{aligned}
& \hat{x}_{1}\left(t_{i+1}\right)=\hat{x}_{1}\left(t_{i}\right)+\left(\hat{x}_{2}\left(t_{i}\right)+\lambda\left|x_{1}\left(t_{i}\right)-\hat{x}_{1}\left(t_{i}\right)\right|^{1 / 2} \operatorname{sign}\left(x_{1}\left(t_{i}\right)-\hat{x}_{1}\left(t_{i}\right)\right)\right) \delta, \\
& \hat{x}_{2}\left(t_{i+1}\right)=\hat{x}_{2}\left(t_{i}\right)+\left(f_{1}\left(t_{i}, x_{1}\left(t_{i}\right), \hat{x}_{2}\left(t_{i}\right), u\left(t_{i}\right)\right)+\alpha \operatorname{sign}\left(x_{1}\left(t_{i}\right)-\hat{x}_{1}\left(t_{i}\right)\right)\right) \delta,
\end{aligned}
$$

where $\hat{x}_{1}\left(t_{i}\right), \hat{x}_{2}\left(t_{i}\right)$ are the estimated variables.

Theorem 3.2 (Davila et al., 2005): Suppose that the function $f_{1}$ is uniformly bounded and condition (11) holds. Then the observation algorithm (13) with parameters (12) ensures the convergence of the estimation errors to the domain $\left|\tilde{x}_{1}\right| \leq \gamma_{1} \delta^{2},\left|\tilde{x}_{2}\right| \leq \gamma_{2} \delta$ where $\gamma_{1}, \gamma_{2}$ are some constants, depending on the observer parameters.

This theorem is proved in Davila et al. (2005).

\subsection{State $x_{3}$}

Consider the subsystem with state variable $x_{3}=v_{f}$, in this case an observer will be introduced in order to obtain an equivalent output injection, in the same form that the states $x_{1}$ and $x_{2}$, the dynamic equation of $x_{3}$ could be written as

$$
\begin{aligned}
& \dot{x}_{3}=f_{2}\left(t, x_{3}, u\right)+\xi_{2}\left(t, x_{2}, x_{3}, u\right) \\
& y_{2}=x_{3},
\end{aligned}
$$

in this case, the dynamic of the system is considered as unknown $\xi_{2}\left(t, x_{2}, x_{3}, u\right)=$ $\frac{C_{x}}{m}-\frac{C_{x} R_{e}}{m} \frac{x_{2}}{x_{3}}$ in consequence $f_{2}\left(t, x_{3}, u\right)=0$. The system (14), understood in Filippov's sense (Filippov, 1988) is assumed such that the perturbation $\xi\left(t, x_{1}, x_{2}, u\right)$ is Lebesgue-measurable and uniformly bounded in any compact region of the state space.

Our task is then to design a finite-time convergent observer of the linear velocity $x_{3}$. The proposed sliding mode observer is given by

$$
\dot{\hat{x}}_{3}=z_{3}
$$

where $z_{3}=\beta \operatorname{sign}\left(x_{3}-\hat{x}_{3}\right)$. Defining $\tilde{x}_{3}=x_{3}-\hat{x}_{3}$, the dynamic of the error for $x_{3}$ becomes

$$
\dot{\tilde{x}}_{3}=\xi_{2}\left(t, x_{2}, x_{3}, u\right)-\beta \operatorname{sign}\left(\tilde{x}_{3}\right)
$$

where $\beta$ is chosen such that $\beta>\max \left(\frac{C_{x}}{m}-\frac{C_{x} R_{e}}{m} \frac{x_{2}}{x_{3}}\right)=\eta$. 
Theorem 3.3: Suppose that $\left|\dot{x}_{3}\right| \leq \eta$, and the parameter $\beta$ is chosen such that $\beta>\eta$. The observer (15) guarantees the convergence of the estimated state $\left(\hat{x}_{3}\right)$ to the real value of the states $\left(x_{3}\right)$ after a finite time transient, and there exists a time constant $t_{1}$ such that for all $t \geq t_{1}, \hat{x}_{3}=x_{3}$.

Proof: Consider the Lyapunov function

$$
V\left(\tilde{x}_{3}\right)=\frac{1}{2} \tilde{x}_{3}^{2}
$$

its time derivative

$$
\dot{V}\left(\tilde{x}_{3}\right)=\tilde{x}_{3} \dot{\tilde{x}}_{3}=\tilde{x}_{3}\left(\xi_{2}\left(t, x_{2}, x_{3}, u\right)-\beta \operatorname{sign}\left(\tilde{x}_{3}\right)\right)
$$

If $\beta$ is chosen as was given in the theorem (3.3), then $\dot{V}\left(\tilde{x}_{3}\right)<0$. This shows that $\tilde{x}_{3}$ goes to zero in a finite time, then, there exist a constant $t_{1}$ such that for all $t \geq t_{1}$ holds $\tilde{x}_{3}=0$

\section{Equivalent output injection analysis}

For the time $t_{2}$ where $t_{2}=\max \left(t_{0}, t_{1}\right)$ and for all $t \geq t_{2}$ the error dynamics (10) and (16) holds

$$
\begin{aligned}
& \dot{\tilde{x}}_{2}=0=F\left(t, x_{1}, x_{2}, \hat{x}_{2}\right)-\alpha \operatorname{sign}\left(\tilde{x}_{1}\right) \\
& \dot{\tilde{x}}_{3}=0=\xi_{2}\left(t, x_{2}, x_{3}, u\right)-\beta \operatorname{sign}\left(\tilde{x}_{3}\right) .
\end{aligned}
$$

Notice in equation (18) that at this time $\hat{x}_{2}=x_{2}$ and $f_{1}\left(t, x_{1}, x_{2}, u\right) \equiv f_{1}\left(t, x_{1}, \hat{x}_{2}, u\right)$ in consequence $F\left(t, x_{1}, x_{2}, \hat{x}_{2}\right)=\xi_{1}\left(t, x_{1}, x_{2}, u\right)$.

It was assumed that the terms $z_{2}, z_{3}$ change at a high (infinite) frequency. However, in reality, various imperfections make the state oscillate in some vicinity of the intersection and components of $z_{2}, z_{3}$ are switched at finite frequency, this oscillations have high and slow frequency components.

The high frequency terms $z_{2}, z_{3}$ are filtered out and the motion in the sliding mode is determined by the slow components Utkin et al. (1999). It is reasonable to assume that the equivalent control is close to the slow component of the real control which may be derived by filtering out the high-frequency component using low pass filter.

The filter time constant should be sufficiently small to preserve the slow components undistorted but large enough to eliminate the high frequency component.

Thus the conditions $\tau \rightarrow 0$ where $\tau$ is the filter time constant, and $\delta / \tau \rightarrow 0$, where $\delta$ is the sample interval, fulfilled to extract the slow component equal to the equivalent control and to filter out the high frequency component.

The above reasons allows us to write the equivalent output injection as

$$
\begin{aligned}
& \bar{z}_{2}=\xi_{1}\left(t, x_{2}, x_{3}, u\right) \\
& \bar{z}_{3}=\xi_{2}\left(t, x_{2}, x_{3}, u\right)
\end{aligned}
$$

where $\bar{z}_{2}$ and $\bar{z}_{3}$ are the filtered versions of $z_{2}$ and $z_{3}$ respectively. 


\section{System identification}

Assuming that $J$ and $m$ are known, and defining $a_{1}=\frac{1}{J}$, it is possible to write the system (6) as follows

$$
\begin{aligned}
& \dot{x}_{1}=x_{2} \\
& \dot{x}_{2}=a_{1} u+\vartheta_{1} \varphi_{1} \\
& \dot{x}_{3}=\vartheta_{2} \varphi_{2} \\
& y=\left[\begin{array}{cc}
x_{1} & 0 \\
0 & x_{3}
\end{array}\right]
\end{aligned}
$$

with

$$
\begin{aligned}
& \varphi_{1}(x)=\left[\begin{array}{c}
\frac{-1}{J} \\
\frac{x_{2}}{J x_{3}}
\end{array}\right] \text { and } \varphi_{2}(x)=\left[\begin{array}{c}
\frac{1}{m_{x_{2}}} \\
-\frac{x_{x_{3}}}{m}
\end{array}\right] \\
& \vartheta_{1}=\left[R_{e} C_{x} R_{e}^{2} C_{x}\right] \text { and } \vartheta_{2}=\left[C_{x} R_{e} C_{x}\right]
\end{aligned}
$$

Notice equation (22) is in a regression form with regressor vectors $\varphi_{1}(x), \varphi_{2}(x)$ in equation (23), and parameters vectors $\vartheta_{1}, \vartheta_{2}$ in equation (23).

Using the regression notation of equation (22) the observer (8) could be written as

$$
\begin{aligned}
& \dot{\hat{x}}_{1}=\hat{x}_{2}+z_{1} \\
& \dot{\hat{x}}_{2}=a_{1} u+\bar{\vartheta}_{1} \varphi\left(t, x_{1}, x_{2}, u\right)+z_{2}
\end{aligned}
$$

where $\bar{\vartheta}_{1}$ is a parameters vector with nominal values of $\vartheta_{1}$. For all $t \geq t_{2}$ equations (20), (21) become

$$
\begin{aligned}
& \bar{z}_{2}=\Delta \vartheta_{1} \varphi_{1}\left(t, x_{1}, x_{2}, u\right) \\
& \bar{z}_{3}=\vartheta_{2} \varphi_{2}\left(t, x_{2}, x_{3}, u\right)
\end{aligned}
$$

where $\Delta \vartheta_{1}=\vartheta_{1}-\bar{\vartheta}_{1}$.

To proceed we will consider, for clarity of presentation only, the estimation procedures in two steps, one for $x_{2}$ and one for $x_{3}$ in order to estimate respectively $\vartheta_{1}$ and then $\vartheta_{2}$.

\subsection{Identification of $\vartheta_{1}$}

It is possible to apply a dynamic form of the Least Square identification algorithm to estimate the parameter vector with the knowledge of $\bar{z}_{2}$ the regression vector deduced from the measurements and observations of $\varphi_{1}$.

The model structure for the linear regression (Davila et al., 2006; Goodwin and Sin, 1984; Soderstrom and Stoica, 1989) can be written as in equation (25) where $\bar{z}_{2}$ is a measurable quantity, $\varphi_{1}(t)$ is a regression vector made of known quantities and $\Delta \vartheta$ is the unknown parameters vector (difference to the nominal parameters). 
The application of linear regression algorithms like the Least Squares parameter estimation algorithm can be written as

$$
\begin{aligned}
& \bar{z}_{2}=\widehat{\Delta \vartheta} \varphi_{1}\left(t, x_{1}, x_{2}, u\right) \\
& \varepsilon(t)=h(t)-\hat{h}(t)=\widetilde{\Delta \vartheta} \varphi_{1}\left(t, x_{1}, x_{2}, u\right) \\
& \dot{\widehat{\Delta \vartheta}}=\frac{\sigma}{\gamma_{t}} \Gamma_{t} \varphi_{1}\left(t, x_{1}, x_{2}, u\right) \varepsilon(t)=\Gamma_{t} \varphi_{1} \varphi_{1}^{T} \widetilde{\Delta \vartheta} \\
& \dot{\Gamma}_{t}=-\frac{\sigma}{\gamma_{t}} \Gamma_{t} \varphi_{1}^{T}(t) \varphi_{1}(t) \Gamma_{t}
\end{aligned}
$$

where $\widehat{\Delta \vartheta}$ is the estimation of $\Delta \vartheta$ the parameters vector and $\hat{h}(t)$ the prediction of the signal $h(t)$. In general $\gamma_{t}$ is a normalisation term $\gamma_{t}=1+\varphi_{1}(t) \Gamma_{t} \varphi_{1}^{T}(t)$ and $\sigma \in[0.9,1]$ a forgetting factor. The initial conditions of the RLS algorithm are $\Gamma_{0}=\rho^{-1} I$ initial gain matrix and $\widehat{\Delta \vartheta}=\widehat{\Delta \vartheta_{0}}$ initial parameters values.

Theorem 5.1 (Davila et al., 2006): The algorithm (27) ensure the following upper bound for the estimation error:

$$
\|\Delta \vartheta\|^{2} \leq n m_{2} \frac{\left(1-\alpha^{t}\right) 2}{(\ln \alpha) 2}\left(\frac{\delta \sqrt{m_{2}}}{|\ln \alpha|}\right)^{2}\|\Gamma(t)\|^{2}
$$

where

$$
\|\dot{\vartheta}(t)\| \leq \delta, \quad\|\sigma\|=\alpha<1, \quad\left\|\varphi(t) \varphi^{T}(t)\right\|=\|\varphi(t)\|^{2} \leq m_{2} .
$$

Remark 5.2: The use of equations (27) ensures the asymptotic convergence of $\widehat{\Delta \vartheta}$ to $\Delta \vartheta$ under the persistent excitation condition (Soderstrom and Stoica, 1989; Goodwin and Sin, 1984).

Remark 5.3: In application, we have considered the delta operator for approximation of the derivation (Goodwin and Sin, 1984).

\subsection{Identification of $\vartheta_{2}$}

The low frequency components of the signal $z_{3}$ satisfies equation (26), using the notation in equation (22) takes the form

$$
\bar{z}_{3}=\beta \overline{\operatorname{sign}}\left(\tilde{x}_{3}\right)=\vartheta_{2} \varphi_{2}=\frac{\vartheta_{21}}{m}-\vartheta_{22} \frac{1}{m} \frac{x_{2}}{x_{3}} .
$$

Remark 5.4: In the same way $\vartheta_{21}$, assuming $\vartheta_{22}$ known or already estimated, can be identified using the Least Squares algorithm.

Remark 5.5: Note also that both parameters in $\vartheta_{2}$ can be estimated by the Least Square Algorithm at this step. This correspond to estimating twice $\vartheta_{22}$, assuming at this step as previous estimation the value produced by the previous step.

Remark 5.6: Note also that depending on the expression formulated for the forces and wheel slip in equations (4), (5) several their variables can be estimated like adherence or longitudinal forces. 


\section{Experimental results}

In this section, we present some experimental results to validate our approach. Several trials have been done with a vehicle (P406 of LCPC, Figure 3) equipped with sensors for wheels angular position measurement (see Figures 3 and 4).

Measures have been acquired with the vehicle rolling at several speeds. The experimental data used here are those of the rear wheel drive. The installed sensors at each wheel are the variable reluctance ones of the Antilock Braking System (ABS see Figure 4). Their resolution is 29 dot per revolution (i.e., $\theta(i)=\frac{2 \pi n(i)}{29} \mathrm{rad}$ ). An additional encoder (with 1000 dot by turn i.e., $\theta(i)=\frac{2 \pi n(i)}{1000} \mathrm{rad}$ ) have been installed for angular position measurements control and validation.

Figure 3 Vehicle used for experiments (see online version for colours)

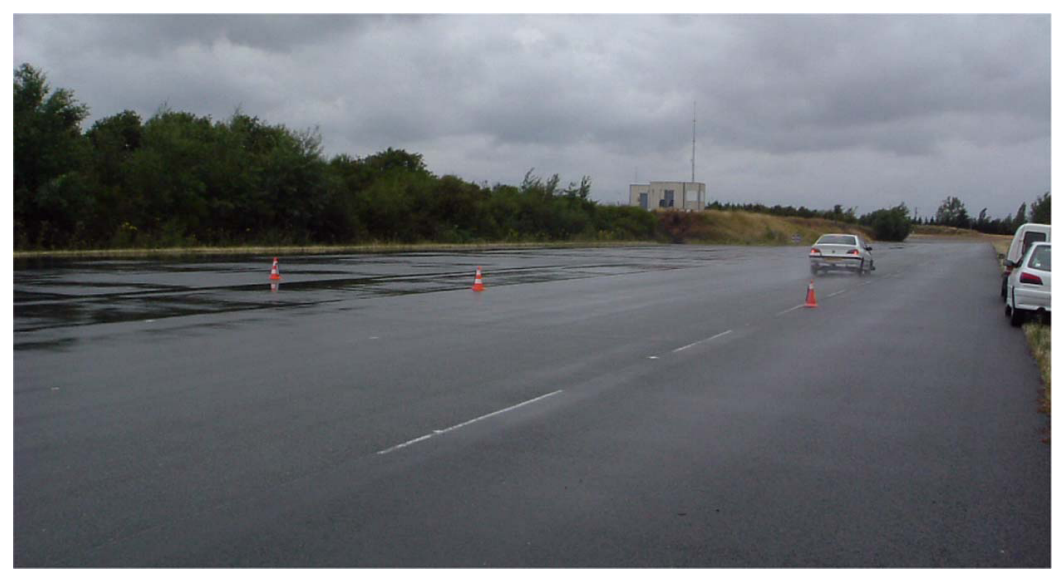

Figure 4 Four sensors used by ABS $29 \operatorname{dot} / 2 \pi$ (see online version for colours)

ABS with 4 Channels

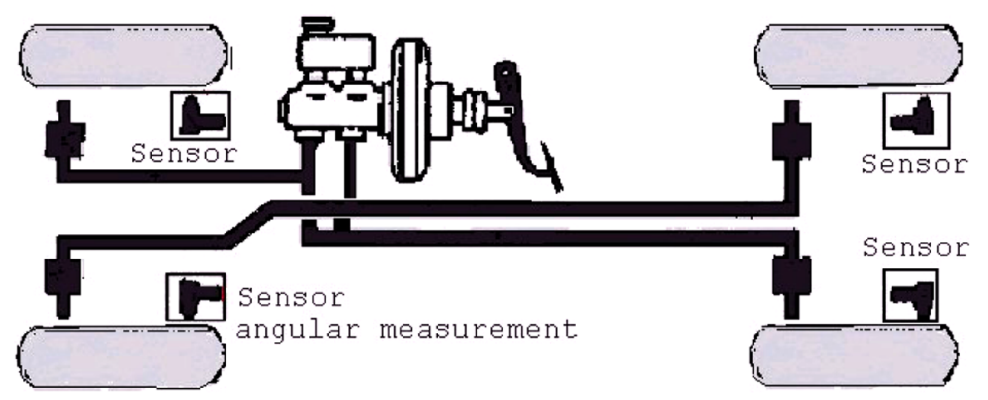

Figure 5 shows the installed laser sensor used for measurement of the wheel radius. Data are sampled at $1 \mathrm{kHz}$ frequency and several trials have been considered a different running speeds $(40,60,80,100 \mathrm{~km} / \mathrm{h}$ and varying velocity) with and without using the ABS system.

Figure 6 shows the measured displacement using the (high and low resolution) sensors installed on the vehicle and the observed one. We can remark that the curve are well superposed despite resolution. 
Figure 5 Sensor for wheel radius measurement (see online version for colours)

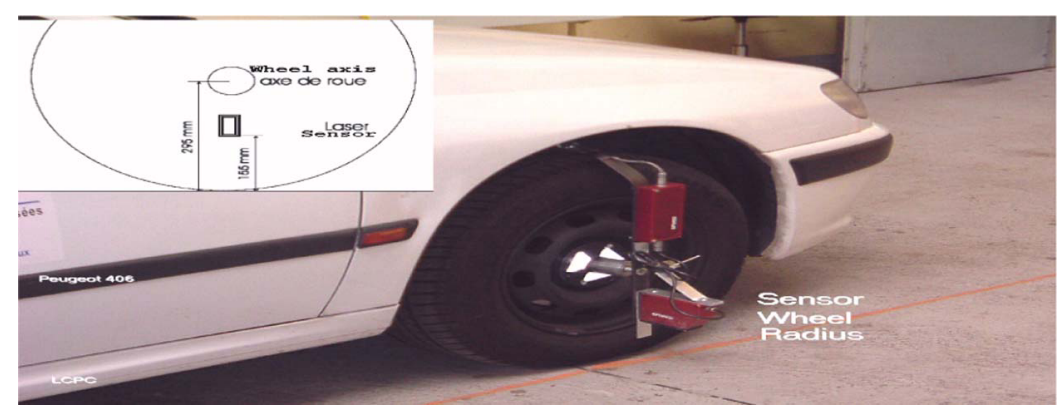

Figure 6 Measured angular displacements (see online version for colours)
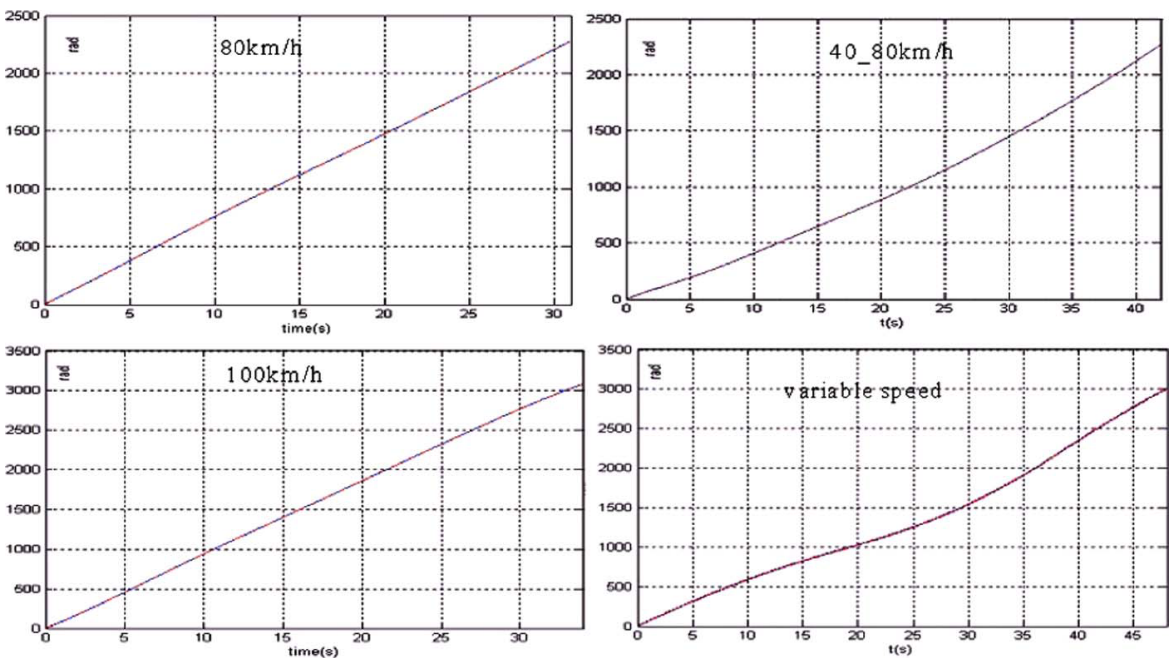

The velocities can be deduced by several ways from the displacement measurements. Here we compare three of them, two standard computation of derivatives and the proposed observer:

$$
\begin{aligned}
& \dot{\theta}(i)=\frac{\theta(i)-\theta(i-1)}{T} \\
& \dot{\theta}(i)=\frac{\theta(i+1)-\theta(i-1)}{2 T} \\
& \hat{x}_{2}=\operatorname{observed}(\dot{\theta}) .
\end{aligned}
$$

In the upper left and right of Figure 7 we can see that estimation of velocity signal derivation, using (31) and (32) respectively, needs a filter to reduce the noise effect. In Figure 8 corresponding to low resolution encoders the problem is worse and amplitude of noise has a higher level. Filtering this data will affect the measurement precision.

We remark that when using the proposed observer (bottom left curve in the two figures) that the estimation remain precise despite the bad resolution of the sensors used by ABS. The observed and reconstructed velocities are compared to the measure provided by a high resolution encoder. 
Figure 7 Estimated velocities using the high sensors $(1000 \mathrm{dot} / \mathrm{t})(\mathrm{see}$ online version for colours)

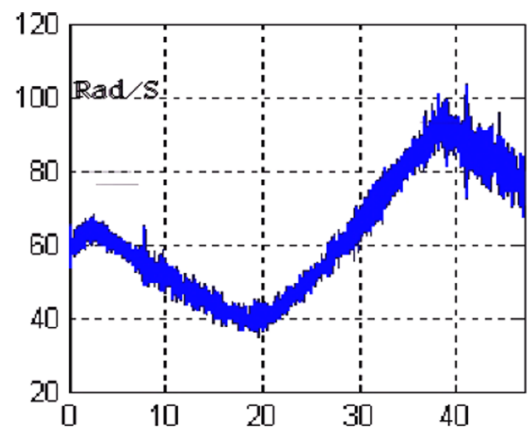

$\mathrm{t}(\mathrm{s})$

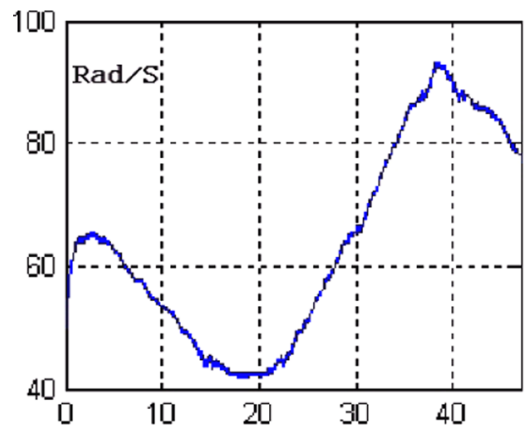

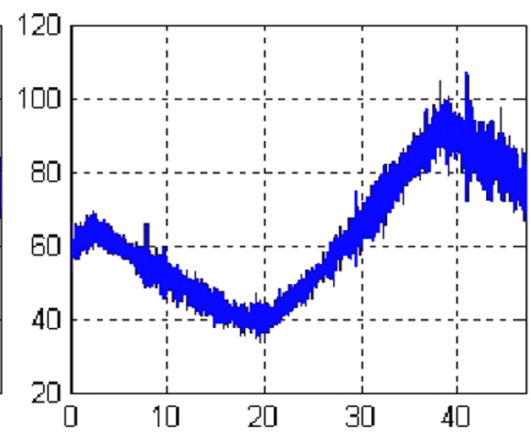

$t(s)$

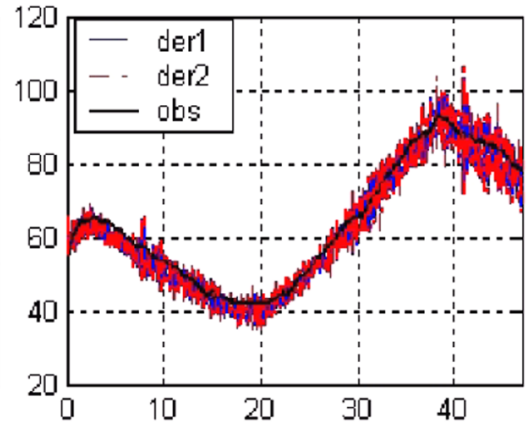

These curves show the robustness of our observer based on second order sliding modes and super twisting algorithm vs. measurement noise and additional perturbations. Recall that the term $\xi\left(t, x_{1}, x_{2}, u\right)=\varphi_{1}(z) \vartheta_{1}$ is not known and correspond to a perturbation to be rejected in a first step; thank to the finite time convergence. In a second step (after the convergence time) this perturbation is retrieved by use of a low pass filtering and them the parameters $\vartheta_{1}$ can be estimated. The second step estimations are the wheel radius and its longitudinal equivalent stiffness. The estimations are shown in Figures 9 and 10.

The estimated parameters are quite good and the algorithm is very easy to apply and is not difficult to tune its parameters.

\section{Conclusion}

The super-twisting second-order sliding-mode algorithm is modified in order to design a velocity observer for vehicle using only the ABS sensors already placed in standard vehicles nowadays. The finite time convergence of the observer is proved and consequently the separation principle can be considered as avoided. The gains of the proposed observer are chosen very easily ignoring the system parameters. This observer is compared, using experimental data, to classical derivation methods and is proven robust despite the bad resolution of the encoders. Its robustness combined with a sliding mode estimation of the vehicle velocity allow us to reconstruct the wheel sleep. In this way, the observability problems are avoided by means of cascaded finite time 
converging observer instead of additional sensors. It can be shown that contact forces can also be estimated by this way.

Figure 8 Estimated velocities using the ABS sensors (29 dot/t) (see online version for colours)
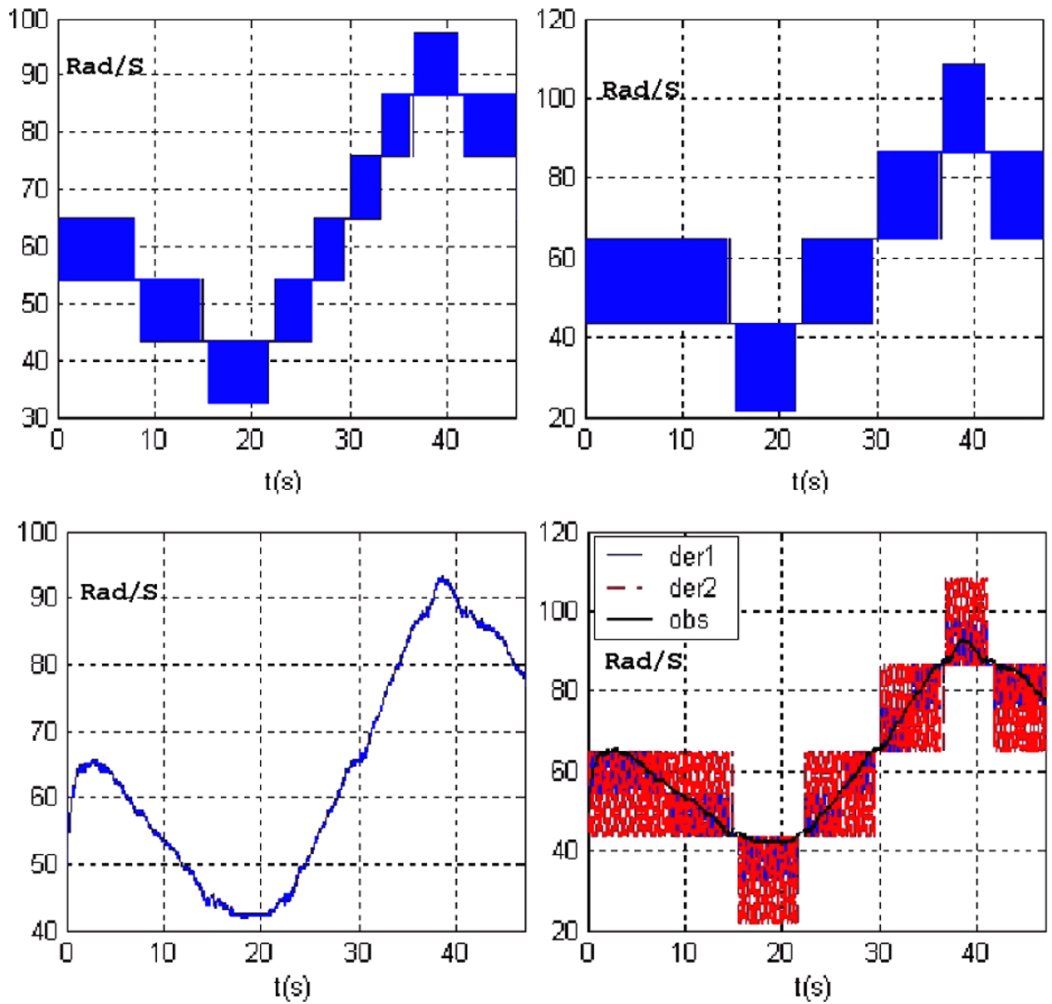

Figure 9 Estimated velocity using the ABS sensors (see online version for colours)
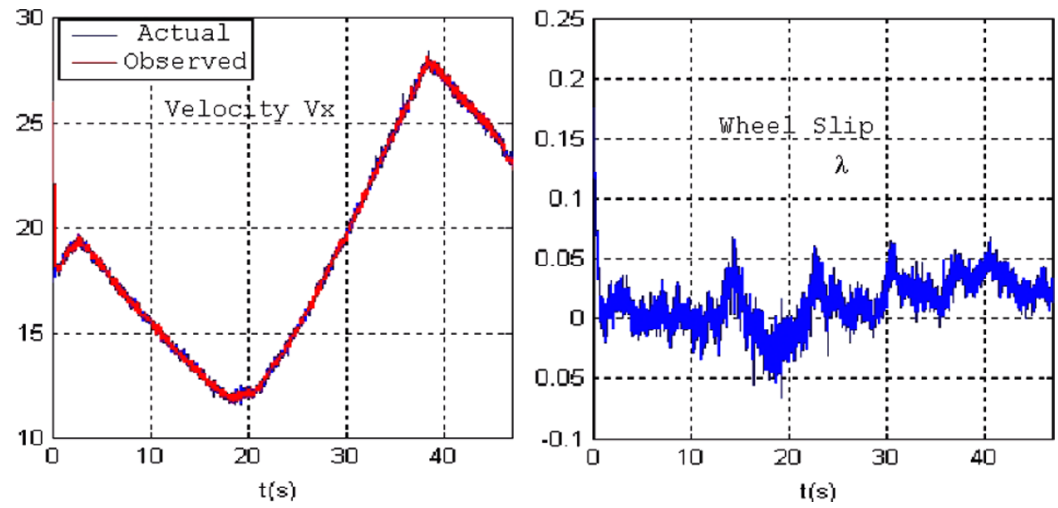

The finite time convergence of state observations in the same time as robustness and perturbation rejection allows to solve the problem of parameter identification using the equivalent control method (by retrieval of the rejected signal). The use of the equivalent control, which provides a linear regression model, allows to apply the 
classical parameter identification methods (RLS) to estimate the systems dynamic parameters like the tyre longitudinal equivalent stiffness and the effective wheel radius.

Figure 10 Estimated wheel stiffness and radius using the ABS sensors and observations
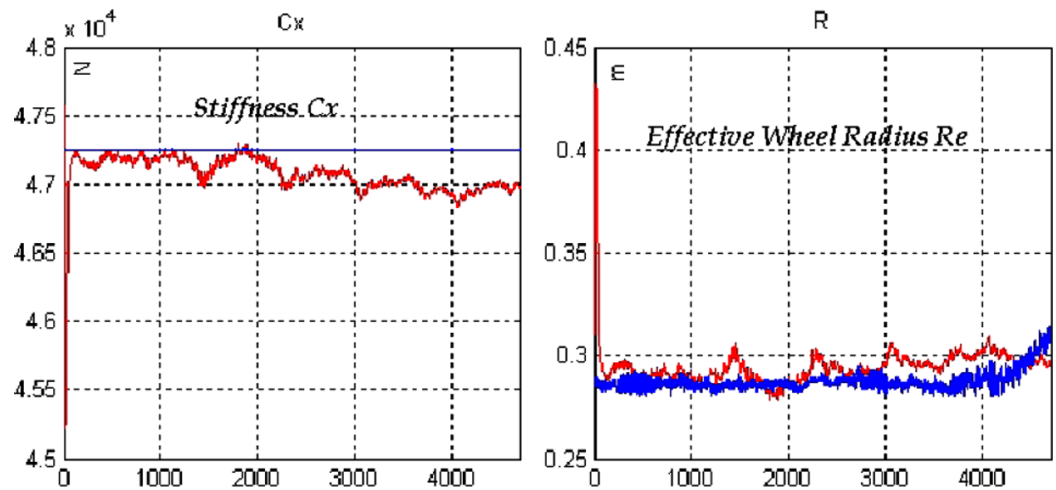

The estimation scheme build up using a Second Order Sliding Mode observers and a Sliding Mode velocity estimator has been tested on experimental data (acquired with a P406 vehicle) and shown to be very efficient using only standard sensors. The actual results prove effectiveness and robustness of the proposed method. In our further investigations we consider also the case of complete vehicle in a road with changing adherence. The estimations produced online will be used to define a predictive control to enhance the safety.

\section{Acknowledgements}

J. Davila and L. Fridman gratefully acknowledge the financial support of the Mexican CONACyT (Consejo Nacional de Ciencia y Tecnologia), Grant No. 56819, the Programa de Apoyo a Proyectos de Investigacion e Innovacion Tecnolgica (PAPIIT) UNAM, grant no. 111208, as well as, DGSCA - DTD. This work has been done in a collaboration managed by members of the LSIS inside the GTAA(Groupe Thématique Automatique et Automobile). The GTAA is a research group supported by the CNRS. Thanks are addressed to the LCPC of Nantes for experimental data and the trials with their vehicle Peugeot 406.

\section{References}

Ackermann, J. (1997) 'Robust control prevents car skidding', IEEE Control Systems Magazine, Vol. 17, No. 3, pp.23-31.

Alvarez, J., Orlov, Y. and Acho, L. (2000) 'An invariance principle for discontinuous dynamic systems with application to a coulomb friction oscillator', Journal of Dynamic Systems, Measurement, and Control, Vol. 122, pp.687-690.

Atassi, A.N. and Khalil, H.K. (2000) 'Separation results for the stabilization of nonlinear systems using different high-gain observer design', Systems and Control Letters, Vol. 39, pp.183-191. 
Bakker, E., Pacejka, H.B. and Linder, L. (1989) 'A new tire model with an application in vehicle dynamics studies', SAE 89, Vol. 98, No. 6, pp.101-113.

Barbot, J.P., Djemai, M. and Boukhobza, T. (2002) 'Sliding mode observers', in Perruquetti, W. and Barbot, J.P. (Eds.): Sliding Mode Control in Engineering, Ser. Control Engineering, No. 11, Marcel Dekker, New York, pp.103-130.

Bartolini, G., Pisano, A., Punta, E. and Usai, E. (2003) 'A survey of applications of second-order sliding mode control to mechanical systems', International Journal of Control, Vol. 76, pp.875-892.

Canudas de Wit, C., Tsiotras, P., Velenis, E., Basset, M. and Gissinger, G. (2003) 'Dynamic friction models for road/tire longitudinal interaction', Vehicle Syst. Dynamics, Vol. 39, No. 3, pp.189-226.

Clover, C.L. and Bernard, J.E. (1998) 'Longitudinal tire dynamics', Vehicle System Dynamics, Vol. 29, pp.231-259.

Carlson, C.R. and Gerdes, C.J. (2003) 'Nonlinear estimation of longitudinal tire slip under several driving conditions', Proceedings of the American Control Conference, June.

Davila, J., Fridman, L. and Levant, A. (2005) 'Second-order sliding-mode observer for mechanical systems', IEEE Trans. Automat. Contr., Vol. 50, No. 11, November, pp.1785-1789.

Davila, J., Fridman, L. and Poznyak, A. (2006) 'Observation and identificatin of mechanical systems via second order sliding modes', Int. J. of Control, Vol. 79, No. 10, pp.1251-1262.

Drakunov, S., Ozguner, U., Dix, P. and Ashrafi, B. (1995) 'ABS control using optimum search via sliding modes', IEEE Trans. Control Systems Technology, Vol. 3, March, pp.79-85.

Dugoff, P.F.H. and Segel, L. (1970) 'An analysis of tire traction properties and their influence on vehicle dynamic performance', SAE Transaction, Vol. 3, pp.1219-1243.

Filippov, A.F. (1988) Differential Equations with Discontinuous Right-hand Sides, Dordrecht, Kluwer Academic Publishers, The Netherlands.

Gim, G. and Nikravesh, P. (1990) 'Analytical model of pneumatic tyres for vehicle dynamic simulations part 1: pure slips', Int. J. Vehicle Design, Vol. 11, No. 6, pp.589-618.

Goodwin, C.G. and Sin, K.S. (1984) Adaptive Filtering Prediction and Control, Prentice-Hall, New Jersey.

Gustafsson, F. (1997) 'Slip-based tire-road friction estimation', Automatica, Vol. 33, No. 6, pp.1087-1097.

Harned, J., Johnston, L. and Scharpf, G. (1969) 'Measurement of tire brake forces characteristics as related to wheel slip (Antilock) control system design', SAE Trans., Vol. 78, pp.909-925.

Ingram, R.W. (1978) 'An investigation of the information content of (certain) social responsibility disclosures', Journal of Accounting Research, Vol. 16, No. 2, pp.270-285.

Kazemi, R., Kabganian, M. and Modir Zaare, M.R. (2000) 'A new (ABS)', Proc. 2000 SAE Automotive Dynamics and Stability Conference, Troy, Michigan, May, pp.263-270.

Lee, H. and Tomizuka, M. (2003) 'Adaptative vehicle traction force control for intelligent vehicle highway systems (IVHSs)', IEEE Trans. on Industrial Electronics, Vol. 50, No. 1, February.

Levant, A. (1993) 'Sliding order and sliding accuracy in sliding mode control', International Journal of Control, Vol. 58, pp.1247-1263.

Levant, A. (1998) 'Robust exact differentiation via sliding mode technique', Automatica, Vol. 34, No. 3, pp.379-384.

Livingston, D.I. and Brown, J.E. (1970) 'Physics of the slipping wheel, II: slip under both travtive and lateral forces', Rubber Chemistry and Technology, Vol. 43, No. 2.

Ljung, L. and Soderstrom, J. (1983) System Identification, MIT Press, Cambridge. 
M'Sirdi, K.N., Rajaoarisoa, L.H. and Naamane, A. (2008) 'Modélisation, Identifiabilité et Observabilité pour le diagnostic et la commande d'une classe de Systèmes Complexes à Structure Variable', in Lefebvre, D., Chafouk, H., El Hami, A. and Bennouna, M. (Eds.): Diagnostic des Systèmes Complexes, ISBN 9954-8992, pp.83-93.

M'Sirdi, N.K., Rabhi, A., Zbiri, N. and Delanne, Y. (2004) 'VRIM: vehicle road interaction modelling for estimation of contact forces', Accepted for TMVDA 04, 3rd Int. Tyre Colloquium Tyre Models For Vehicle Dynamics Analysis, University of Technology, Vienna, Austria, 30-31 August.

M'Sirdi, N.K., Rabhi, A. and Naamane, A. (2007) 'Vehicle models and estimation of contact forces and tire road friction', ICINCO-RA, Vol. 1, pp.351-358.

Pacejka, H.B. and Besseling, I. (1997) 'Magic formula tyre model with transient properties', 2nd International Tyre Colloquium on Tyre Models for Vehicle Dynamic Analysis, Swets and Zeitlinger, Berlin, Germany.

Pisano, A. and Usai, E. (2004) 'Output-feedback control of an underwater vehicle prototype by higher-order sliding modes', Automatica, Vol. 40, pp.1525-1531.

Rabhi, A., Imine, H., M'Sirdi, N. and Delanne, Y. (2004a) 'Observers with unknown inputs to estimate contact forces and road profile', AVCS'04 International Conference on Advances in Vehicle Control and Safety, Genova-Italy, October, pp.28-31.

Rabhi, A., M'Sirdi, N.K., Zbiri, N. and Delanne, Y. (2004b) Modélisation Pour L'estimation de l'état et des Forces d'Interaction Véhicule-Route, Soumis au CIFA, Douz, Tunisie.

Rabhi, A. et al. (2003) 'Modélisation des forces de contact véhicule-chaussée', Rapport interne: LRV, UVSQ, 10, avenue de l'Europe 78140 Vélizy, France, rabhi@lrv.uvsq.fr

Ray, L. (1995) 'Nonlinear state and tire force estimation for advanced vehicle control', IEEE T on Control Systems Technology, Vol. 3, No. 1, March, pp.117-124.

Samadi, B. and Nikravesh, K.Y. (2001) 'Estimation of vehicle speed and tire-road friction forces', Amirkabir Journal of Science and Technology, Vol. 12, No. 46, Spring, pp.237-245.

Shtessel, Y.B., Shkolnikov, I.A. and Brown, M.D.J. (2003) 'An asymptotic second-order smooth sliding mode control', Asian Journal of Control, Vol. 5, No. 4, pp.498-504.

Sira-Ramirez, H. (2002) 'Dynamic second-order sliding mode control of the hovercraft vessel', IEEE Trans. on Contr. System Technology, Vol. 10, pp.860-865.

Soderstrom, T. and Stoica, P. (1989) System Identification, Prentice-Hall International, Cambridge, Great Britain.

Spicer, B.H. (1978) 'Investors, corporate social performance and information disclosure: an empirical study', The Accounting Review, Vol. 53, No. 1, pp.94-111.

Utkin, V., Guldner, J. and Shi, J. (1999) Sliding Mode Control in Electromechanical Systems, Taylor \& Francis, London, UK. 